\title{
Aprendizaje Servicio en el Máster Iberoamericano en Cooperación Internacional y Desarrollo: una experiencia de innovación docente
}

\section{Gutiérrez Cruz, Alexandra y Sierra Rodríguez, Belinda*}

*Cátedra de Cooperación Internacional y con Iberoamérica de la Universidad de Cantabria (gcruza@unican.es, sierrab@unican.es)

\begin{abstract}
This paper aims to systematize the objectives, educational process and results of the "Project Service-Learning (hereinafter APS) on Cooperation for Development", a proposal for educational innovation developed in the Iberoamerican Master in International Cooperation and Development (MICID) of the University of Cantabria (UC) during the academic year 2014-2015.

In the first section, it is described the educational context in which the proposal was developed and pedagogically justified the relevance of implementing the methodology of APS in MICID. In order to do this, it is emphasized the educational and transformative potential of this strategy as a tool that allows an experiential and meaningful learning. Besides, APS can generate knowledge located and encourages the development of necessary and desirable competences in Cooperation for Development, not only from the declarative and procedural field ("knowledge" and "know-how") but also and above all, psychological ("knowing how to be" and "knowing how to behave"). In the second section, it is described the objective of APS and systematized the planning, development and evaluation process of this innovative teaching proposal. Finally, in the last section, the main results and learnings obtained are described (from the point of view of students, the training organization and the participating institutions).
\end{abstract}

Keywords: Service-Learning, competences, training graduate, International Cooperation, Human Development, Educational Innovation, active pedagogy. 
Aprendizaje Servicio en el Máster Iberoamericano en Cooperación Internacional y Desarrollo: una experiencia de innovación docente

\begin{abstract}
Resumen
La presente comunicación tiene como objetivo sistematizar los objetivos, proceso pedagógico y resultados del "Proyecto Aprendizaje Servicio (ApS) en Cooperación para el Desarrollo", una propuesta de innovación docente desarrollada en el Máster Iberoamericano en Cooperación Internacional y Desarrollo (MICID) de la Universidad de Cantabria (UC) durante el curso académico 2014-2015.
\end{abstract}

En el primer apartado, se describe el contexto educativo en que se desarrolla la propuesta y se justifica pedagógicamente la pertinencia de implementar la metodología del ApS en el MICID. Para ello, se subraya el potencial educativo y transformador de esta estrategia, en tanto que herramienta que facilita un aprendizaje vivencial y significativo, permite generar saberes situados y favorece el desarrollo de competencias necesarias y deseables en cooperación para el desarrollo, desde el ámbito no solo declarativo y procedimental ("saber" y "saber hacer") sino también y, sobre todo, psicosocial ("saber ser" y "saber estar"). En el segundo apartado, se describe el objetivo de la propuesta de ApS y se sistematiza el proceso de planificación, desarrollo y evaluación de dicha experiencia de innovación docente. Por último, en el tercer apartado, se describen los principales resultados y aprendizajes obtenidos (desde el punto de vista del alumnado, de la entidad formativa y de las instituciones participantes).

Palabras clave: Aprendizaje Servicio, competencias, formación de posgrado, Cooperación Internacional, Desarrollo Humano, Innovación Docente.

\title{
1. Motivaciones, justificación y contexto de la propuesta de Aprendizaje Servicio en el MICID
}

El Máster Iberoamericano en Cooperación Internacional y Desarrollo (MICID) de la Universidad de Cantabria (UC) nace, hace nueve años, con el objetivo de ofrecer una formación de calidad, que permita a sus egresados desempeñarse con excelencia en los diferentes ámbitos de promoción del desarrollo humano, tanto en sede como en terreno.

Se trata de un título propio, de carácter profesionalizante, que cuenta desde 2012 con el reconocimiento del Consejo de Acreditación Internacional de Estudios del Desarrollo e Investigación (IAC/EADI), una mención que reconoce la calidad de su labor en la formación de profesionales del desarrollo.

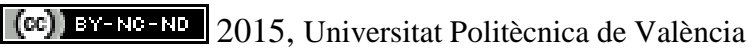


El equipo de dirección y docencia del MICID, consciente de los retos que plantea la formación universitaria de posgrado, y con el objetivo de contribuir al desarrollo de las nuevas competencias profesionales que exige la Cooperación Internacional para el Desarrollo Humano (CID), inicia en 2011 un proceso de reflexión para repensar y reorientar su programa formativo, el cual afecta tanto a los contenidos, como a las estrategias de enseñanza-aprendizaje.

Entre los procesos de innovación docente más destacados en las últimas ediciones del MICID, se encuentra la inclusión de la metodología Aprendizaje Servicio (ApS) durante el presente curso académico 2014-2015. Se trata de una estrategia de enseñanza-aprendizaje que, si bien no es nueva, ha comenzado a generalizarse en España en los últimos años en el ámbito educativo, siendo numerosos los estudios e investigaciones que confirman el impacto positivo que tiene el ApS en el ámbito universitario en España (Francisco y Moliner, 2010; Folgueiras, Luna y Puig, 2013; Rodríguez Gallego, 2014, entre otros).

No existe en la actualidad una definición única y consensuada sobre el término de ApS, encontrándose matices muy distintos en las diferentes aproximaciones conceptuales a esta metodología. No obstante, hay consenso en considerar como nucleares y definitorios dos elementos. El primero, el aprendizaje de contenidos conceptuales, procedimentales y actitudinales ligados al currículo, en nuestro caso, a los objetivos y competencias formativas del MICID; el segundo, el servicio o labor voluntaria para transformar un entorno concreto y/o dar respuesta a necesidades reales.

De igual modo, son rasgos característicos del ApS los siguientes (basado en Puig et al., 2009):

- La coordinación y trabajo en red con la comunidad (entidades sociales, centros educativos, administraciones, asociaciones, empresas, etc.).

- La respuesta a necesidades reales.

- Una pedagogía basada en la experiencia real vivida por el alumnado.

- El aprendizaje en valores, el compromiso ético y el uso del conocimiento como vía para transformar contextos y para ofrecer soluciones a necesidades y problemáticas concretas.

A modo de síntesis, se presentan dos definiciones que recogen la esencia del ApS, tal y como se ha trabajado en esta propuesta de innovación docente:

- Propuesta educativa que combina procesos de aprendizaje y de servicio a la comunidad en un único proyecto, en el que los participantes aprenden a la vez que trabajan sobre necesidades reales del entorno para mejorarlo (Puig Rovira y Palos (2006). 
Aprendizaje Servicio en el Máster Iberoamericano en Cooperación Internacional y Desarrollo: una experiencia de innovación docente

- Método de enseñanza que potencia tanto el aprendizaje académico que se desarrolla en el aula, como la realización de un servicio voluntario para dar respuesta a las necesidades detectadas en el entorno o comunidad (MartínezOdría, 2007).

La dirección y coordinación del MICID, teniendo en cuenta la evaluación y feedback de los alumnos egresados de las ediciones anteriores, así como los resultados de diferentes estudios sobre competencias profesionales en cooperación para el desarrollo (Gutiérrez et al. 2014, Gutiérrez 2013, Gutiérrez y Garvi 2013, Gutiérrez, 2011 y Tres, 2006), decidió apostar por el ApS en tanto que metodología con potencial pedagógico para facilitar un aprendizaje vivencial y significativo, generar saberes situados y desarrollar competencias necesarias y deseables en cooperación internacional para el desarrollo (CID), desde el ámbito no solo declarativo y procedimental ("saber" y "saber hacer”), sino también, y sobre todo, psicosocial ("saber ser" y "saber estar). En definitiva, se considera que es una metodología, coherente con el enfoque de aprendizaje de competencias, y que servirá para desarrollar contenidos teóricos y especializados sobre CID, facilitar la adquisición de recursos para movilizarlos y ofrecer un contexto real y situado para aplicarlos, en nuestro caso, organizaciones cántabras que trabajan en el ámbito de la cooperación y el desarrollo.

\section{Objetivos y desarrollo de la propuesta de innovación docente}

Como ya se ha señalado, la propuesta de innovación docente consiste en implantar la metodología de ApS en la novena edición del MICID. El fin último es favorecer que el alumnado desarrolle las competencias formativas de este programa de máster mientras realiza un servicio vinculado a la cooperación y el desarrollo. A su vez, se espera que la acción solidaria del alumnado mejore el aprendizaje realizado en el aula, lo dote de sentido y permita extraer nuevos aprendizajes.

La propuesta de ApS se ha desarrollado entre les meses de noviembre de 2014 a junio de 2015.

El proceso pedagógico que la sustenta puede resumirse en seis fases cronológicas (adaptadas de Puig, Martín y Batllé, 2008) 


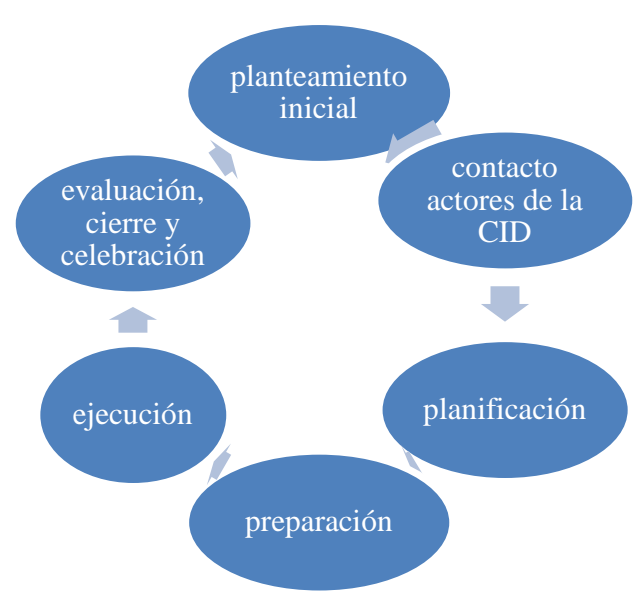

Fig.1 Fases del desarrollo de la propuesta de ApS en el MICID

Para una mayor comprensión, se describen a continuación las principales acciones y tareas llevadas a cabo en cada fase:

\subsection{Planteamiento inicial}

Una vez consensuada la decisión de implementar la innovación metodológica de ApS, se realizó una primera reflexión general sobre la situación de la cooperación para el desarrollo en Cantabria y de sus principales actores, con el objetivo de explorar potenciales escenarios en los que desarrollar el ApS. Para ello, fue muy valioso el conocimiento acumulado y compartido de la Cátedra de Cooperación Internacional y con Iberoamérica de la Universidad de Cantabria (COIBA), actor estratégico de la cooperación cántabra y responsable del MICID. La segunda tarea consistió en identificar los aprendizajes vinculados al programa formativo que querían potenciarse, transferirse y desarrollarse con el ApS. Las competencias psicosociales, relacionadas con el saber ser y saber estar, y las procedimentales (saber hacer), fueron priorizadas sobre las competencias declarativas o cognitivas (saber), al considerarse que estas últimas se trabajan de manera más intensa en el aula.

Tabla 1. Competencias formativas del MICID 
Aprendizaje Servicio en el Máster Iberoamericano en Cooperación Internacional y Desarrollo: una experiencia de innovación docente

\begin{tabular}{|c|c|c|}
\hline $\begin{array}{l}\text { COMPETENCIAS } \\
\text { GENERALES }\end{array}$ & DEFINICIÓN & COMPETENCIAS ES PECIIFICAS \\
\hline $\begin{array}{l}\text { SABER (MODELO } \\
\text { DECLARATIVO) }\end{array}$ & $\begin{array}{l}\text { Conocimiento y } \\
\text { comprensión del } \\
\text { andamiaje } \\
\text { conceptual y teórico } \\
\text { de la cooperación y } \\
\text { el desarrollo } \\
\text { humano }\end{array}$ & $\begin{array}{l}\text { Comprender los principales debates de la Economía del Desarrollo (pobreza, exclusión, desigualdad, } \\
\text { desarrollo humano, globalización, etc.). } \\
\text { Conocer la agenda y sistema internacional del desarrollo: consensos internacionales, arquitectura, actores } \\
\text { y marcos de coperación innovadores. } \\
\text { Comprender el marco institucional, político, normativo y jurídico de la cooperacñión al desarrollo } \\
\text { española. } \\
\text { Identificar los principales factores socioculturales, políticos, antropológicos, psicológicos, demográficos } \\
\text { y económicos que influyen en el desarrollo. }\end{array}$ \\
\hline $\begin{array}{l}\text { SABER HACER } \\
\text { (PROCEDIMENTAL) }\end{array}$ & $\begin{array}{l}\text { Manejo de las } \\
\text { habilidades y } \\
\text { destrezas necesarias } \\
\text { para ap licar dichos } \\
\text { conocimientos }\end{array}$ & $\begin{array}{l}\text { Manejar las principales fuentes de información sobre cooperación internacional y desarrollo humano. } \\
\text { Manejar las nuevas tecnologías de la información y la comunicación, las redes sociales, el networking } \\
\text { las herramientas necesarias para la adecuada gestión del conocimiento sobre el desarrollo. } \\
\text { Demostrar competencia para la planificación estratégica de procesos de desarrollo a largo plazo. } \\
\text { Demostrar habilidad en el uso de las técnicas y herramientas necesarias para la gestión del ciclo del } \\
\text { proyecto. } \\
\text { Ser capaz de emitir informes de asesoramiento y realizar trabajos de consultoría (notas conceptuales, } \\
\text { dictámenes, posicionamientos) sobre cuestiones relacionadas con el desarrollo humano (en su nivel } \\
\text { internacional, nacional o descentralizado). } \\
\text { Demostrar competencia para la interlocución interinstitucional, la incidencia política y el cabildeo. } \\
\text { Demostrar capacidad pedagógica y competencia para la comunicación eficaz con público profesional y } \\
\text { no profesional. } \\
\text { Ser capaz de trabajar en un equipo multidisciplinar y multicultural y de compartir el conocimiento } \\
\text { profesional. }\end{array}$ \\
\hline $\begin{array}{l}\text { SABER SER Y ESTAR } \\
\text { (PSICOSOCIAL) }\end{array}$ & $\begin{array}{l}\text { Interiorización y } \\
\text { apropiación de los } \\
\text { valores y actitudes } \\
\text { de la cooperación y } \\
\text { el desarrollo } \\
\text { humano }\end{array}$ & $\begin{array}{l}\text { Demostrar una actitud intercultural y empática, aceptando la diversidad de soluciones para promocionar } \\
\text { el desarrollo. } \\
\text { Asumir un enfoque de agencia individual, mediación y facilitacion en los procesos de desarrollo. } \\
\text { Ser creativo e innovador en la búsqueda de soluciones relacionadas con el desarrollo, dadas unas } \\
\text { problemáticas v recursos concretos. } \\
\text { Tener capacidad para adaptarse a personas y a contextos nuevos. } \\
\text { Demostrar rigor, consistencia y pensamiento crítico en los análisis sobre los problemas del desarrollo. } \\
\text { Ser responsable, riguroso, proactivo y orientado al aprendizaje permanente. } \\
\text { Demostrar compromiso ético con la promoción del desarrollo humano e interiorización de los principios } \\
\text { y valores de la cooperación. }\end{array}$ \\
\hline
\end{tabular}

\subsection{Contacto con actores de la cooperación para el desarrollo en Cantabria}

La selección de las instituciones y entidades sociales para la realización del servicio se realizó atendiendo a tres criterios:

a) Coherencia con la Responsabilidad Social de la Universidad (RSU): la entidad o institución en que se realiza el APS ha de ser una entidad sin ánimo de lucro, orientada a servicios de interés general y con una misión, visión y valores coherentes con los principios de la RSU y el Desarrollo Humano.

b) Ámbito de trabajo: Su campo de actuación ha de estar relacionado con la cooperación internacional y la promoción del desarrollo humano. 
c) Grado de interés y motivación en el proyecto de APS: Dicho criterio sirvió para descartar aquellas entidades interesadas únicamente en la realización de prácticas, voluntariado, becas o trabajo de campo.

De acuerdo con estos criterios las entidades participantes fueron las siguientes:

Tabla 2. Organizaciones participantes en el Aprendizaje Servicio (ApS) del MICID 2014/2015

\begin{tabular}{|c|c|c|}
\hline ORGANIZACIÓN & TIPO ACTOR & MISIÓN \\
\hline $\begin{array}{l}\text { Fondo Cantabria Coopera } \\
\text { (FCC) }\end{array}$ & $\begin{array}{l}\text { Fundación } \\
\text { Pública }\end{array}$ & $\begin{array}{l}\text { Promover una cooperación descentraliza de calidad } \\
\text { dando preferencia a la cooperación técnica en la } \\
\text { variante de asistencia técnica pública (cooperación } \\
\text { directa), a la educación para el desarrollo y a la } \\
\text { responsabilidad social corporativa. }\end{array}$ \\
\hline $\begin{array}{l}\text { Ayudar a Despegar de } \\
\text { Cantabria (ADEC) }\end{array}$ & ONGD & $\begin{array}{l}\text { Promover el Desarrollo Humano y Sostenible en } \\
\text { Melong II (Camerún). }\end{array}$ \\
\hline $\begin{array}{l}\text { Área de Cooperación } \\
\text { Internacional para el } \\
\text { Desarrollo (ACOIDE) de la } \\
\text { Universidad de Cantabria }\end{array}$ & Universidad & $\begin{array}{l}\text { Promover en la UC una cooperación de calidad } \\
\text { orientada al desarrollo humano y sostenible, con el } \\
\text { fin último de contribuir a la eliminación de las } \\
\text { desigualdades internacionales y a la erradicación } \\
\text { de la pobreza en el mundo. }\end{array}$ \\
\hline $\begin{array}{l}\text { Red Española de Estudios } \\
\text { del Desarrollo (REEDES) }\end{array}$ & Asociación & $\begin{array}{l}\text { Promover la investigación básica y aplicada, la } \\
\text { formación interdisciplinar, y la calidad de éstas, en } \\
\text { los estudios sobre y para el desarrollo. }\end{array}$ \\
\hline $\begin{array}{l}\text { Asociación } \\
\text { Nueva Vida }\end{array}$ & $\begin{array}{l}\text { Asociación / } \\
\text { ONGD }\end{array}$ & $\begin{array}{l}\text { Acción social integral dirigida a la inclusión de } \\
\text { reclusos y ex reclusos, víctimas de violencia, } \\
\text { víctimas de tráfico y explotación sexual, personas } \\
\text { en situación de dependencia, colectivos en gran } \\
\text { necesidad y/o exclusión; y el desarrollo en países } \\
\text { con menores oportunidades mediante proyectos de } \\
\text { cooperación. }\end{array}$ \\
\hline $\begin{array}{l}\text { Brigadas Internacionales de } \\
\text { Paz (PBI) }\end{array}$ & ONGD & $\begin{array}{l}\text { Defensa y promoción de los derechos humanos, } \\
\text { ofreciendo asistencia y apoyo a proyectos } \\
\text { internacionales en Colombia, Guatemala, México y } \\
\text { Nepal. }\end{array}$ \\
\hline Medicus Mundi Cantabria & $\begin{array}{l}\text { Asociación / } \\
\text { ONGD }\end{array}$ & $\begin{array}{l}\text { Generar cambios en la sociedad, fomentando una } \\
\text { cultura de solidaridad y compromisos ciudadanos, } \\
\text { que hagan posible la erradicación de la pobreza y } \\
\text { permitan que la salud sea un derecho al alcance de } \\
\text { todas las personas. }\end{array}$ \\
\hline
\end{tabular}

\subsection{Planificación}

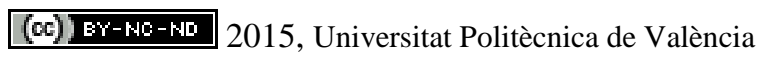

Congreso In-Red (2015) 
Aprendizaje Servicio en el Máster Iberoamericano en Cooperación Internacional y Desarrollo: una experiencia de innovación docente

La asignación de las y los alumnos a las organizaciones se realizó utilizando como criterio el grado de ajuste entre las expectativas y perfiles (personal y profesional) del alumnado y las características, misión, visión y ámbito de trabajo de las entidades. Para ello se entrevistó a los responsables de cada institución, explicándose los objetivos del $\mathrm{ApS}^{1}$ e identificándose posibles líneas de acción y servicio. A su vez, se envió una encuesta al alumnado que sirvió para conocer sus expectativas sobre el ApS, las áreas temáticas vinculadas a la cooperación al desarrollo que más despertaban su interés y las restricciones y limitaciones horarias para desarrollar el proyecto.

El siguiente paso consistió en la elaboración de la rúbrica de evaluación. Para ello se tuvieron en cuenta los criterios y recomendaciones del Grup de Recerca en Educació Moral de la Facultat de Pedagogía de la UB (2014).

\subsection{Preparación}

El objetivo principal de esta fase fue el de motivar al grupo, describir los objetivos, metodología y evaluación del ApS y comunicar a cada alumno la entidad o institución asignada para la realización del ApS. El 24 de febrero de 2015 se celebró una sesión presencial que sirvió para justificar pedagógicamente la pertinencia del ApS en el MICID e informar al alumnado sobre los principales aspectos organizativos, didácticos y de evaluación.

Posteriormente, las instituciones participantes, el alumnado participante y COIBA (como responsable y coordinadora del MICID) firmaron un acuerdo de colaboración, que recogía los objetivos del ApS, así como las obligaciones y derechos de cada parte.

\subsection{Ejecución}

Cada proyecto de APS ha tenido una duración de 100 horas y ha sido desarrollado entre los meses de marzo, abril y mayo de 2015. Dado que el horario lectivo del Máster es de 16.00 horas a 20.00 horas, de lunes a jueves, la mayoría del alumnado ha dedicado las mañanas de dichos días a la ejecución del proyecto.

La primera tarea realizada por el alumnado consistió en la aproximación general a la entidad en que participaría y la identificación de las necesidades, problemáticas y oportunidades de actuación. Tras este proceso de investigación -reflexión, cada alumno/a,

\footnotetext{
${ }^{1}$ Posteriormente se envió a cada entidad documentación y bibliografía básica cobre ApS para profundizar en esta metodología.
} 
según el aprendizaje realizado en el MICID, sus conocimientos previos y su motivación personal, formularon un proyecto de APS, haciendo constar:

- Nombre del proyecto y definición breve y clara del servicio

- Nombre de la entidad y de las personas comprometidas

- Breve diagnóstico de la necesidad y finalidad del servicio

- Personas destinatarias

- Objetivos tangibles del servicio

- Potenciales aprendizajes

- Descripción de las acciones o actividades

- Entidad o entidades sociales que participan

- Organización y calendario de trabajo

Durante los tres meses de ejecución del ApS, la coordinación del MICID ha estado en contacto con el alumnado y con las instituciones participantes para hacer un seguimiento de su trabajo y resolver las incidencias y dudas que pudieran surgir. A su vez, cada alumno/a recibió un Diario Personal para documentar y sistematizar las vivencias, aprendizajes y reflexiones sobre el ApS.

En total, se han desarrollado 9 proyectos. Atendiendo a la tipología de ApS propuesta por Martínez (2008), la mayoría de las iniciativas desarrolladas se clasifican dentro de la categoría "servicio directo", y de manera más concreta, se vinculan con la transferencia de conocimiento en materia de cooperación y desarrollo humano a los actores de la cooperación cántabra (planificación, formulación según Enfoque de Marco Lógico, sensibilización y Educación para el Desarrollo, comunicación institucional, gestión de redes sociales, etc.). No obstante, tal y como como comprobarse tras el cruce de contenido de las Tablas 1 y 2, la mayoría de los proyectos incluyen elementos propios de las categorías, "servicio de investigación” y, en menor medida, de "advocacy".

Tabla 1. Resumen de modelos de proyectos de APS (basado en Martínez 2008). 
Aprendizaje Servicio en el Máster Iberoamericano en Cooperación Internacional y Desarrollo: una experiencia de innovación docente

\begin{tabular}{|c|c|}
\hline vicio & $\begin{array}{l}\text { yectos en los que el alumnado interviene directamente sobre una necesidad } \\
\text { creta, interactuando directamente con las personas o instituciones implicadas } \\
\text { dicha realidad. }\end{array}$ \\
\hline & $\begin{array}{l}\text { Proyectos trabajados desde la institución educativa, esto es desde el MICID. Se } \\
\text { trabaja sobre una determinada problemática o necesidad pero sin trabajar de } \\
\text { manera directa con las personas afectadas o con las instituciones comunitarias } \\
\text { que intentan dar una respuesta. }\end{array}$ \\
\hline ddve & $\begin{array}{l}\text { Proyectos orientados a eliminar las causas que mantienen o perpetúan una } \\
\text { problemática o una necesidad no satisfecha. Son típicas las acciones que tienen } \\
\text { que ver con la movilización social, la sensibilización o la incidencia política para } \\
\text { transformar una determinada situación. }\end{array}$ \\
\hline vicio de & $\begin{array}{l}\text { ara analizar una } \\
\text { ta. }\end{array}$ \\
\hline
\end{tabular}

Tabla 2. Resumen de proyectos de ApS realizados durante el MICID 2014/2015 


\begin{tabular}{|c|c|c|}
\hline $\begin{array}{l}\text { PROCEDENCIA } \\
\text { GEOGRÁFICA Y } \\
\text { TITULACIÓN DEL } \\
\text { ALUMNO/A }\end{array}$ & ORGANIZACIÓN & $\begin{array}{c}\text { TÍTULO DEL PROYECTO DE } \\
\text { APS }\end{array}$ \\
\hline $\begin{array}{l}\text { Honduras / Lic. en Admón. } \\
\text { de Empresas }\end{array}$ & FCC & $\begin{array}{l}\text { Propuesta de Cooperación Técnica } \\
\text { en el Instituto Genaro Muñoz Diseñar un proyecto de Cooperación Técnica para ser } \\
\text { Hernández del Municipio de implementado en Honduras. } \\
\text { Siguatepeque (Honduras) }\end{array}$ \\
\hline EE.UU / Lic. en Economía & ADEC & $\begin{array}{l}\text { Proyecto básico de producción } \\
\text { agrícola para el sostenimiento de la Formulación de un proyecto de cooperación en Camerún } \\
\text { casa de la Oportunidad (Melong una convocatoria pública de subvenciones. } \\
\text { II, Camerún) }\end{array}$ \\
\hline $\begin{array}{l}\text { Honduras / Lic. en } \\
\text { Contaduría Pública y } \\
\text { Finanzas }\end{array}$ & $\begin{array}{l}\text { Asoc. Evangélica } \\
\text { Nueva Vida }\end{array}$ & 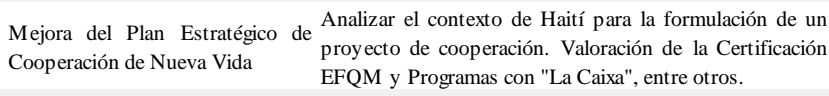 \\
\hline España / Lic. en Derecho & REEDES & $\begin{array}{ll}\text { Mejora de la incidencia política y } & \begin{array}{l}\text { Diseñar una estrategia de incidencia política. Gestión de } \\
\text { las redes sociales. Asistencia técnica en la Ley Orgánica de }\end{array} \\
\text { pública de REEDES } & \text { Protección de Datos. }\end{array}$ \\
\hline $\begin{array}{l}\text { España / Ing. de Caminos, } \\
\text { Canales y Puertos }\end{array}$ & $\begin{array}{l}\text { Asoc. Evangélica } \\
\text { Nueva Vida }\end{array}$ & $\begin{array}{ll}\text { Mejora del Plan Estratégico de } & \begin{array}{l}\text { Analizar el contexto de Haití para la formulación de un } \\
\text { proyecto de cooperación. Valoración de la Certificación }\end{array} \\
\text { Cooperación de Nueva Vida } & \text { EFQM y Programas con "La Caixa", entre otros. }\end{array}$ \\
\hline $\begin{array}{l}\text { Argentina / Lic. en Ciencia } \\
\text { Política }\end{array}$ & $\begin{array}{l}\text { Medicus Mundi } \\
\text { Cantabria }\end{array}$ & $\begin{array}{l}\text { Analizar las actividades de la ONGD, así como su relación } \\
\begin{array}{l}\text { Análisis del tejido asociativo de con socios y voluntarios. Mejorar la visibilidad de la } \\
\text { Medicus Mundi Cantabria }\end{array} \\
\begin{array}{l}\text { organización a través de la creación de perfiles en las redes } \\
\text { sociales. }\end{array}\end{array}$ \\
\hline $\begin{array}{l}\text { Paraguay / Lic. en Rel. } \\
\text { Internacionales }\end{array}$ & $\begin{array}{l}\text { Fondo Cantabria } \\
\text { Coopera }\end{array}$ & $\begin{array}{l}\text { Propuesta de Cooperación Técnica } \\
\text { con el Centro Educativo Diseñar un proyecto de Cooperación Técnica para ser } \\
\text { Mbaracayu (Paraguay) }\end{array}$ \\
\hline $\begin{array}{l}\text { México / Lic. en Rel. } \\
\text { Internacionales }\end{array}$ & ACOIDE & $\begin{array}{l}\begin{array}{l}\text { Dinamización del voluntariado Gestión de la convocatoria de voluntariado internacional. } \\
\text { gestionado y promovido por Diagnóstico del voluntariado que está haciendo un grupo } \\
\text { ACOIDE }\end{array} \quad \text { de investigacion de la Universidad de Cantabria. }\end{array}$ \\
\hline España / Lic. en Geografía & PBI & $\begin{array}{l}\text { Modernización y mejora de los Gestión del área de comunicación y transparencia de la } \\
\text { instrumentos de visibilización de entidad. Apoyo en la formulación de proyectos, así como } \\
\begin{array}{ll}\text { PBI } & \text { en otras fases de gestión del ciclo del proyecto. }\end{array}\end{array}$ \\
\hline
\end{tabular}

\subsection{Evaluación, cierre y celebración}

Una vez finalizados los proyectos de APS se envió por correo electrónico la rúbrica de evaluación al alumnado y a las instituciones participantes. Dicha rúbrica tenía como objetivo valorar el servicio prestado, las competencias desarrolladas, los aprendizajes realizados y otros aspectos generales del proceso.

Una vez codificadas y analizadas las respuestas, la dirección y coordinación del MICID redactó un informe final que recogía los principales resultados, recomendaciones finales y lecciones aprendidas. Dicho documento será socializado el 24 de junio de $2015^{2}$ en un

${ }^{2}$ Esta fecha es posterior al momento en que se redacta la presente comunicación

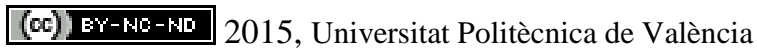

Congreso In-Red (2015) 
Aprendizaje Servicio en el Máster Iberoamericano en Cooperación Internacional y Desarrollo: una experiencia de innovación docente

encuentro en el que participarán las instituciones y entidades participantes, el alumnado y la dirección y coordinación del MICID. El objetivo de esta sesión es el de visibilizar y reconocer públicamente los proyectos realizados, hacer una valoración crítica del proceso de ApS, identificar lecciones aprendidas y celebrar los aprendizajes adquiridos.

\section{Resultados}

Los resultados preliminares de la experiencia, a falta de la sesión de cierre y de celebración, se han organizado en dos apartados. En el primero se muestran los resultados relacionados con los aspectos generales del proceso de APS. En el segundo, se describen los aprendizajes y competencias desarrollados mediante el proyecto de APS.

\subsection{Valoración general del proceso de APS}

En términos generales, se destaca la valoración positiva del ApS, por parte tanto del alumnado, como del equipo directivo del MICID y de los diferentes actores de la CID que han participado. Así, lo expresan los comentarios generales realizados por los responsables (R) y los alumnos (A):

- "Como primera experiencia (en ApS), me ha parecido muy buena. Con la sesión final de presentación conjunta, creo que quedará muy redondo (R).

- "El ApS me ha ayudado mucho para aplicar lo que hemos visto en clase en la realidad" (A).

- "He aprendido desde aplicación de conceptos y metodologías que vi en clase de forma teórica, hasta curiosidades generales de países donde la entidad trabaja, hay un abanico muy grande de lecciones que me llevo) (A).

- $\quad$ "La puesta en marcha del ApS ha supuesto romper con inercias y automatismos en la docencia, potenciar la motivación por la innovación y poner en valor la excelencia académica del MICID”. (R)

Se detallan a continuación los aspectos pedagógicos, organizativos e institucionales analizados para valorar la experiencia y calidad del proceso de APS. 
Tabla 3. Criterios de evaluación del proceso de ApS (basados en Grup de Recerca en Educació Moral de la Facultat de Pedagogía de la UB (2014). 
Aprendizaje Servicio en el Máster Iberoamericano en Cooperación Internacional y Desarrollo: una experiencia de innovación docente

\begin{tabular}{|c|c|c|}
\hline \multirow{2}{*}{ CRITERIO } & \multicolumn{2}{|l|}{ NIVELES } \\
\hline & 2 & 3 \\
\hline 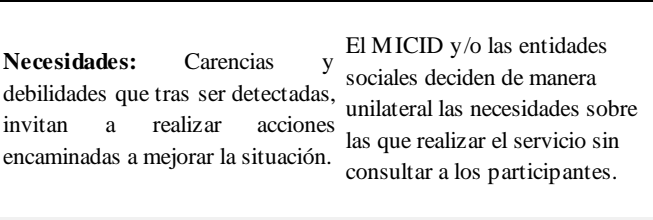 & $\begin{array}{l}\text { Los particip antes junto con el } \\
\text { M ICID o la entidad social, } \\
\text { deciden las necesidades sobre } \\
\text { las que quieren actuar, a través } \\
\text { del análisis de diferentes } \\
\text { problemáticas y la elección de } \\
\text { una de ellas. }\end{array}$ & $\begin{array}{l}\text { Los alumnos descubren de } \\
\text { manera autónoma las } \\
\text { necesidades y debilidades tras } \\
\text { conocer en profundidad la } \\
\text { entidad social y su contexto. }\end{array}$ \\
\hline $\begin{array}{ll}\text { Servicio: Conjunto de tareas que } & \begin{array}{l}\text { Servicio de corta duración } \\
\text { compuesto por tareas sencillas }\end{array} \\
\text { se llevan a cabo contribuy endo a } \\
\text { mejorar alguna situación o paliar } \\
\text { cuya realización supone una } \\
\text { exigencia e implicación }\end{array}$ & $\begin{array}{l}\text { Servicio que permite adquirir } \\
\text { experiencia y destreza en la } \\
\text { realización de tareas de } \\
\text { complejidad media. }\end{array}$ & $\begin{array}{l}\text { Servicio compuesto por tareas } \\
\text { complejas que los propios } \\
\text { participantes deben diseñar } \\
\text { para resolver un problema que } \\
\text { exige creatividad, implicando un } \\
\text { elevado compromiso del } \\
\text { alumno. }\end{array}$ \\
\hline $\begin{array}{l}\text { Sentido del Servicio: Apunta al Servicio que da respuesta a una } \\
\text { impacto de la actividad realizada, necesidad no detectada por el } \\
\text { bien por la utilidad que aporta, o alumno y del que los } \\
\text { bien por el compromiso que participantes no perciben su } \\
\text { manifiestas los protagonistas. } \quad \text { utilidad. }\end{array}$ & $\begin{array}{l}\text { Servicio que da respuesta a una } \\
\text { necesidad de cuya utilidad o } \\
\text { dimensión social los } \\
\text { participantes son conscientes. }\end{array}$ & $\begin{array}{l}\text { Los alumnos dan respuesta a } \\
\text { una necesidad, son conscientes } \\
\text { de su utilidad pero perciben los } \\
\text { límites del servicio si no es } \\
\text { sostenible o mantenido en el } \\
\text { tiempo. }\end{array}$ \\
\hline $\begin{array}{l}\text { Aprendizaje: Adquisición } \\
\text { espontánea o promovida por los Los aprendizajes no están } \\
\text { educadores de conocimientos, } \\
\text { programados, se adquieren de } \\
\text { competencias, conductas y valores. }\end{array}$ & $\begin{array}{l}\text { Los aprendizajes están } \\
\text { relacionados con el programa } \\
\text { del MICID. }\end{array}$ & $\begin{array}{l}\text { El APS sirve para desarrollar } \\
\text { competencias formativas del } \\
\text { MICID. }\end{array}$ \\
\hline
\end{tabular}

Los particip antes se limitan a

Participación: Intervención que realizar las tareas que llevan a cabo los implicados en una previamente otros han actividad con la intención de programado para el desarrollo contribuir junto con otros actores, de la actividad, sin la a su diseño, aplicación y posibilidad de introducir evaluación. modificaciones a la propues
m inicial.

Los participantes realizan aportaciones puntuales

requeridas y/o comparten la responsabilidad en el diseño y desarrollo del conjunto de la actividad.

Proceso de trabajo interdependiente entre

Trabajo en Grupo: Proceso de ayuda entre iguales que se dirige a Procesos espontáneos de ayuda participantes en un proyecto
la preparación y desarrollo de una entre participantes que realizan colectivo que requiere articular $\begin{array}{lll}\text { la preparación y desarrollo de una entre participantes que realizan } & \text { colectivo que requiere articular } \\ \text { actividad que se realiza una actividad individual. } & \text { aportaciones complementarias }\end{array}$ conjuntamente. para alcanzar un objetivo común.

Los participantes se convierten en promotores y responsables del proyecto de modo que intervienen en todas sus fases, decidiendo sobre los diferentes aspectos relevantes.

El trabajo colectivo va más allá del grupo inicial de participantes e incorpora de forma activa a otros agentes externos, creando así redes de trabajo.

Reflexión: Mecanismos de La actividad reflexiva no está La reflexión está programada y optimización del aprendizaje, prevista, ni se proponen tareas hay tareas previstas para basado en la consideración de la para impulsarla, aunque de facilitarla, aunque ocupa sólo experiencia vivida para darle modo natural puede pensarse y un tiempo limitado y separado sentido y lograr nuevos someterse a debate la propia del curso de las actividades del conocimientos. $\quad$ experiencia. proyecto.

\begin{tabular}{|c|c|c|c|}
\hline $\begin{array}{l}\text { Reconocimiento: Conjunto de } \\
\text { acciones destinadas a comunicar a } \\
\text { los protagonistas de la actividad } \\
\text { que la han realizado correctamente. }\end{array}$ & $\begin{array}{l}\text { No hay actividades de } \\
\text { reconocimiento previstas, } \\
\text { aunque de manera espontánea } \\
\text { los diferentes agentes que } \\
\text { intervienen pueden agradecer y } \\
\text { valorar la tarea realizada por los } \\
\text { protagonistas. }\end{array}$ & $\begin{array}{l}\text { Los responsables organizan } \\
\text { actividades destinadas a } \\
\text { reforzar positivamente el } \\
\text { trabajo de los participantes y/o } \\
\text { llevan a cabo iniciativas para } \\
\text { expresar su gratitud y celebrar } \\
\text { el éxito del servicio. }\end{array}$ & $\begin{array}{l}\text { El reconocimiento a los } \\
\text { participantes adquiere una } \\
\text { dimensión pública, } \\
\text { visibilizándose su trabajo por } \\
\text { parte del MICID o de la } \\
\text { entidades sociales. }\end{array}$ \\
\hline $\begin{array}{l}\text { Evaluación: Proceso de obtención } \\
\text { de información para conocer el } \\
\text { desempeño de los participantes en } \\
\text { una actividad y ofrecerles un } \\
\text { feedback que les ayude a mejorar. }\end{array}$ & $\begin{array}{l}\text { No existe un plan de evaluación } \\
\text { establecido, aunque los } \\
\text { responsables, de manera } \\
\text { espontánea y puntual, pueden } \\
\text { evaluar y comunicar su } \\
\text { valoración a los participantes. }\end{array}$ & $\begin{array}{l}\text { Los responsables del MICID y } \\
\text { de las entidades sociales ap lican } \\
\text { un plan de evaluación para } \\
\text { valorar el proceso de APS y las } \\
\text { competencias adquiridas. }\end{array}$ & $\begin{array}{l}\text { Los participantes, junto con los } \\
\text { responsables, intervienen de } \\
\text { manera activa en el proceso de } \\
\text { evaluación del APS. }\end{array}$ \\
\hline
\end{tabular}


Como puede observarse en los Gráficos 1 y 2 la puntuación del alumnado y de los responsables es media- alta en todos los criterios analizados.

Gráfico 1

Puntuación media por criterio según alumnado
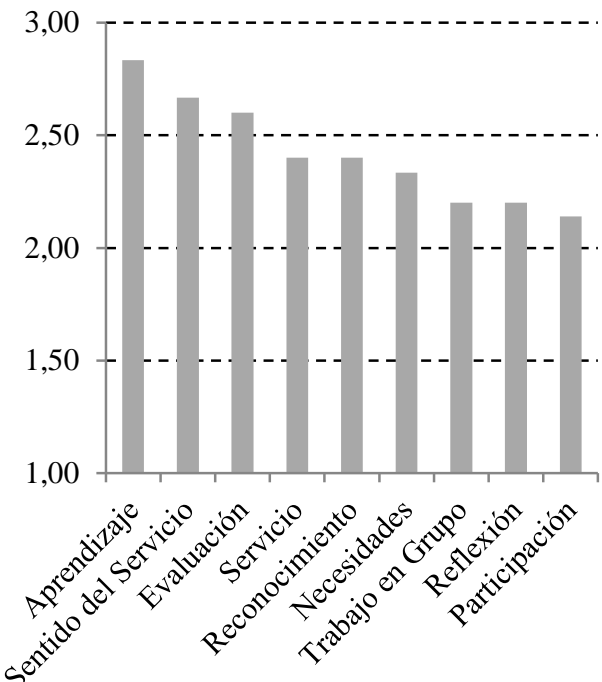

Gráfico 2

Puntuación media por criterio según organizaciones
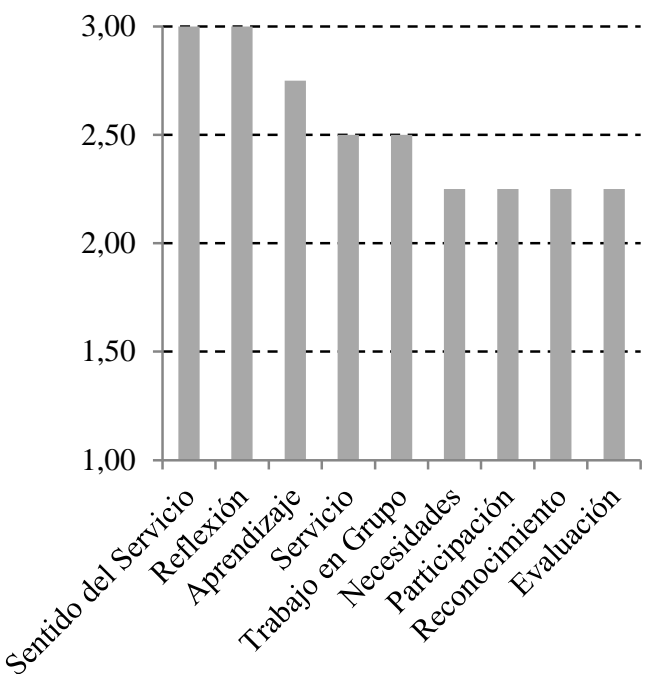

Para el alumnado, la vinculación y coherencia de los aprendizajes realizados con el programa formativo y objetivos competenciales del MICID; el sentido del servicio, esto es la utilidad y prosocialidad del trabajo realizado, y la evaluación participativa, son los tres aspectos mejor valorados del proceso de ApS. Así se observa también en el análisis del contenido de las respuestas cualitativas ofrecidas por el alumnado:

- $\quad$ "De manera informal, los conocimientos adquiridos en la docencia del MICID son afianzados”. (A)

- "Creo que mi trabajo con la entidad es muy útil... y estoy enseñando a la técnica de la entidad el formato también para el futuro. También hemos hablado que yo podría seguir ayudando desde EEUU”. (A)

- "La actividad fue debidamente evaluada por todos los participantes y por la institución promotora.”(A)

(c)) EY-NC-ND 2015, Universitat Politècnica de València

Congreso In-Red (2015) 
Aprendizaje Servicio en el Máster Iberoamericano en Cooperación Internacional y Desarrollo: una experiencia de innovación docente

Por su parte, las organizaciones participantes subrayan los aprendizajes realizados, la reflexión generada, la calidad, sentido y potencial transformador del servicio y la necesidad de dar sostenibilidad a los procesos iniciados con el ApS. Se comprueba, pues, que la puesta en marcha de esta estrategia de enseñanza-aprendizaje ha supuesto la transferencia de conocimiento académico, especializado y de vanguardia a las organizaciones y les ha ofrecido la posibilidad de reflexionar y repensarse:

- El trabajo ha sido de gran importancia para la entidad. De hecho gran, parte del trabajo de los próximos meses se basará en llevar a cabo el plan de incidencia que el alumno ha diseñado”. (R)

- $\quad$ "Se ha dado una respuesta a una necesidad, pero se es consciente de los límites del servicio si no es sostenible”. (R)

- "Para mi autoconocimiento, como técnica de la oficina, de cara a aspectos de mejora, me ha sido de mucha utilidad, porque me ha hecho más consciente de aspectos urgentes de mejora de la gestión de la oficina. (R)

En relación con los comentarios anteriores, se considera que otros de los impactos significativos del ApS han sido el fortalecimiento institucional del MICID y el reconocimiento de su calidad académica, como consecuencia de su apertura a la comunidad y al entorno, del refuerzo del tejido asociativo y de su apuesta por la potenciación del territorio como escenario pedagógico.

Por último, es importante destacar que, según el alumnado, las cuestiones relacionadas con la reflexión han sido las menos valoradas, comparativamente. La revisión y mejora de este criterio se plantea como uno de los principales retos en próximas ediciones, dada la importancia que tiene la reflexión como conector entre los aprendizajes y el servicio.

\subsection{Aprendizajes y competencias}

Tanto el alumnado como los responsables de las entidades coinciden en afirmar que el ApS ha servicio para desarrollar y potenciar las principales competencias procedimentales y psicosociales que inspiran el currículo del MICID. Se reconoce, pues, el valor educativo que tiene para el alumnado, en tanto que permite consolidar los contenidos académicos y teóricos trabajados en el aula y desarrollar competencias y habilidades sociales, emocionales, intelectuales y personales.

En la Tabla 4 se muestra la valoración (de 0 a 3) del alumnado y de los responsables sobre la oportunidad que les ha brindado el ApS para adquirir y desarrollar las competencias procedimentales (“saber hacer”) y psicosociales (“saber ser” y "saber estar”) del MICID. De igual modo, se incluyen algunos ejemplos discursivos significativos que explican el sentido de la puntuación.

(c)) EY-NC-ND 2015, Universitat Politècnica de València

Congreso IN-RED (2015) 
Tabla 4. Valoración de las competencias según el alumnado y los responsables

\begin{tabular}{lcc}
\hline COMPETENCIAS / COMENTARIOS ALUMNADO (A) Y & $\begin{array}{c}\text { Puntuación } \\
\text { Media } \\
\text { Alumnado }\end{array}$ & $\begin{array}{c}\text { Puntuación } \\
\text { Media } \\
\text { Organizaciones }\end{array}$ \\
\hline
\end{tabular}

1-. Demostrar una actitud intercultural, empática, aceptando la diversidad de soluciones para dar respuesta a una problemática.

"Ha cambiado mi percepción de la incorporación de los reclusos en la vida diaria, dándoles apoyo a su reinserción a la vida diaria y laboral" (A), "El alumno ha demostrado su capacidad de entender y adaptarse a diferentes formas de pensar y actuar, así como empatía y cercanía con las realidades expuestas" ( $R)$.

2-. Ser creativo e innovador en la búsqueda de soluciones relacionadas con problemáticas y recursos concretos.

"He creado de cero varios instrumentos que serán de utilidad en el futuro" (A), "Las propuestas de soluciones que ha ofrecido han sido variadas y siempre teniendo en cuenta los limitados recursos de la oficina. Ha sido muy proactivo" (R).

3-. Capacidad de adaptación a personas y contextos nuevos. $\quad 2,67 \quad 2,75$

"Nunca había trabajado con tanta autonomía y (...) ha supuesto un reto de integración para mí" (A), "Su adaptación a la oficina y el equipo de trabajo ha sido muy buena" (R).

4-. Demostrar rigor, consistencia y pensamiento crítico de la realidad en el análisis.

"Al llegar desde fuera tiendes a ver la realidad y las necesidades de un sitio de manera distinta de aquellos de dentro" (A), " Pensamiento crítico no le falta. Es más, le gusta que se le plantee la vuelta de tuerca de las cosas, la otra cara de la moneda. Esta es la mejor forma de aprender a pensar." (R).

5-. Ser responsable, riguroso, proactivo y orientado al aprendizaje permanente.

"He propuesto varias tareas y perspectivas de actuación sobre temas de los que nunca se habían hablado durante la coordinación, así que sí" (A), "Él mismo ha organizado su horario y su ritmo de trabajo. Ha cumplido los plazos y ha dejado sus productos bien presentados y ordenados" (R).

6-. Compromiso ético con la promoción del desarrollo humano e interiorización de los principios y valores de la Cooperación.

"He podido ver como se trabaja con una ética y respetando siempre la privacidad de los beneficiarios." (A), "Precisamente la ética de la cooperación es una gran preocupación del alumno. En su día a día también lo tiene muy presente, y esto se nota y agradece." (R).

7-. Capacidad de trabajar en un equipo multidisciplinar, multicultural y heterogéneo para compartir conocimiento profesional.

"He podido desarrollar una labor en un ambiente muy multicultural y heterogéneo, sin ningún problema, donde cada uno ha podido expresar sus conocimientos con toda naturalidad, a pesar de ser una organización con un carácter evangélico" (A), "Ha compartido conocimiento profesional de anteriores experiencias, buscando la forma de que las buenas prácticas que él conocía se pudieran aplicar aquí, adaptadas" (R).

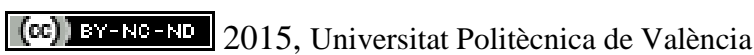

Congreso In-Red (2015) 
Aprendizaje Servicio en el Máster Iberoamericano en Cooperación Internacional y Desarrollo: una experiencia de innovación docente

8-. Capacidad para el acompañamiento, la facilitación de procesos, descubrimiento de capacidades y oportunidades de desarrollo.

"Sí, en gran medida. En este sentido, fue una experiencia muy gratificante." (A), "Buena capacidad" (R).

9-. Habilidades para la comunicación interinstitucional y relaciones interpersonales.

"Sí, no era igual mi manera de dirigirme a mi coordinadora, que a los miembros de la Junta Directiva o al economista Ha Jonn Chang. Hay que adaptarse a cada nivel" (A), "Como en todo trabajo, se pone en marcha esta competencia. No obstante, considero que en otras organizaciones se desarrollará más esta competencia" (R).

10-. Habilidad para la planificación estratégica y gestión del tiempo.

"No fui muy eficaz en la gestión del tiempo. Completamos el $70 \%$ de las actividades propuestas." (A), " Creo que ha desarrollado mucho esta competencia. Él mismo ha organizado su trabajo y sus tiempos, bajo unos lineamientos muy generales." (R).

11-. Capacidad pedagógica y competencia para la comunicación eficaz.

"No tengo mucha capacidad pedagógica. En este caso, lo facilitó la buena predisposición de mi coordinadora." (A), "Ha participado en un taller con estudiantes de $4^{\circ}$ ESO y ha sido una de las actividades que creo que ha disfrutado y donde se le ha sentido cómodo. Sabe conectar con los chavales" (R).

12-. Capacidad para la realización de actividades de consultoría y asistencia técnica.

"El ApS es mi primera experiencia y todavía necesito ayuda para ello" (A), "Han demostrado una alta capacidad como técnicos desde luego contaríamos con ellas como candidatas a nuestros puestos de gestión" (R).

13-. Dominio de nuevas tecnologías de la información y comunicación, uso de redes sociales, gestión del conocimiento y networking.

"Sí y mucho, desde Twitter a Wikipedia" (A), "Ha aportado algunas ideas nuevas en materia de redes sociales que en estamos practicando ya" $(R)$.

14-. Autoconocimiento e identidad: conocimiento de sus potencialidades, limitaciones, fortalezas, aspectos de mejora y responsabilidades.

"La experiencia en general me ha permitido aprender en todos los sentidos, y a tener la disposición de aprender y desaprender" (A).

15-. Conciencia Organizacional: Comprende las características de la entidad con que se trabaja y actuar de manera coherente con su misión y valores.

"Sí, ahora conozco la identidad y el quehacer cotidiano" (A), " Han sabido acomodarse y respetando la identidad de la entidad alinearse con la misma para poder desarrollarse de una manera bastante eficaz" (R). 
Como puede comprobarse, tanto el alumnado como los responsables de las entidades consideran que todas las competencias clave del MICID se vinculan en mayor o menor medida con los aprendizajes y servicio realizado. Se observa, además, que la evaluación de los responsables es más positiva que la autoevaluación realizada por el alumnado. Así, por ejemplo, si bien las organizaciones valoran con la puntuación máxima la capacidad demostrada para la realización de actividades de consultoría y de asistencia técnica, el alumnado percibe que su aportación y habilidad en este ámbito no ha podido desarrollarse lo suficiente con el ApS o ha sido muy modesta, justificándolo por su falta de experiencia profesional o por la corta duración del servicio.

En cualquier caso, el análisis de los comentarios realizados por ambas partes, confirma la coherencia del ApS con el Enfoque de Aprendizaje por Competencias y, en nuestro caso, su pertinencia para favorecer una formación integral en CID. A su vez, se comprueba que los resultados obtenidos son coherentes con los trabajos realizados por muchos otros autores. Así, se confirma la utilidad y conveniencia del ApS para motivar el aprender a aprender y emprender (Martínez,2008); desarrollar el compromiso social y cívico (Martínez Odría, 2007); desarrollar habilidades sociales y reinventar la capacidad crítica (Puig, Batllé, Bosch y Palos, 2007), adquirir conocimientos significativos para la profesión, en este caso como especialistas en cooperación para el desarrollo (Furco y Billig, 2002), desarrollar razonamiento creativo de acuerdo con una base de conocimiento integrada y flexible (Martín, 2009) y estimular el desarrollo del sentido de colaboración para alcanzar una meta común (Halsted, 1998).

\section{Conclusiones}

El MICID es un programa de posgrado vivo, en permanente reflexión y reconstrucción para adaptarse a los cambios y a los nuevos desafíos que plantea la formación universitaria en CID, como son los derivados de la actual sociedad de la información, de la complejización de las estrategias de enseñanza-aprendizaje en la Educación Superior y de la evolución y profesionalización de la cooperación para el desarrollo. La innovación docente aquí presentada viene motivada por el compromiso de su equipo directivo y docente con la sustitución de una enseñanza bancaria centrada en contenidos por un modelo de aprendizaje integral, que favorezca que el alumno aprenda a aprender, se desenvuelva en la actual sociedad del conocimiento y desarrolle su actitud crítica y ética en relación con el desarrollo humano.

La evaluación de la experiencia aquí descrita permite realizar una valoración positiva del potencial pedagógico del ApS para la formación de profesionales de la CID. Se trata de 
Aprendizaje Servicio en el Máster Iberoamericano en Cooperación Internacional y Desarrollo: una experiencia de innovación docente

una estrategia de enseñanza-aprendizaje que ha permitido al alumnado relacionarse con distintos profesionales y actores de la cooperación para el desarrollo, potenciar su compromiso con el Desarrollo Humano y realizar aprendizajes significativos, tanto desde el punto de vista personal como profesional.

Se confirma, por tanto, la posibilidad que brinda el ApS de generar nuevos aprendizajes y de desarrollar competencias relacionadas tanto con la acción, como con el saber ser y saber estar en la profesión. A su vez, se comprueba la motivación que supone para el alumnado la transferencia en forma de acción de los conocimientos adquiridos en el programa del máster, dotando de sentido a los contenidos más teóricos trabajados en el aula.

Los profesionales del desarrollo necesitan unos conocimientos científicos multidisciplinarios que les permitan comprender la complejidad del desarrollo, pero, además, una serie de habilidades procedimentales, personales, sociales y emocionales que les permitan desempeñarse con eficacia, calidad y ética en los distintos ámbitos de promoción del Desarrollo Humano.

Se considera que el APS es una herramienta o metodología que puede dar respuesta a muchos de los desafíos de la actual sociedad del conocimiento y que contribuye a una de las funciones esenciales de la Universidad, a saber, la de formar no sólo profesionales competentes, sino además ciudadanos críticos y comprometidos con la transformación de su entorno en claves de justicia, equidad y desarrollo humano. En nuestro caso, en el ámbito de la CID, comprobamos que el desarrollo del ApS aporta experiencia vital, desarrolla competencias para la vida y para la profesión y potencia la apertura del MICID a su entorno, lo que le ayuda a situarse no sólo como un productor de conocimiento, sino además como generador de "saberes situados" para una acción reflexiva, crítica y ética en materia de desarrollo humano.

El ApS quizás no sea una solución mágica, pero esta experiencia modesta, demuestra que, la Responsabilidad Social Universitaria y la excelencia académica no están reñidas.

\section{Referencias}

FOLGUEIRAS BERTOMEU, P., LUNA GONZÁLEZ, E., y PUIG LATORRE, G. (2013). "Aprendizaje y Servicio: estudio del grado de satisfacción de estudiantes universitarios" en Revista de Educación, 362, p. 159-185.

FRANCISCO AMAT, A., y MOLINER MIRAVE, L. (2010). "El aprendizaje Servicio en la Universidad: una estrategia en la formación de ciudadanía crítica” en Revista Electrónica de formación de profesorado, 13 (4), p. 69-77. 
FURCO, A. y BILLIG, S.H. (Ed.) (2002). Service-Learning: The essency of pedadogy. CT: Information Age, Greenwich.

GRUP DE RECERCA EN EDUCACIÓ MORAL (GREM) (2014). Rúbrica para la autoevaluación y la mejora de los proyectos de APS. $<\underline{\text { http://www.aprenentatgeservei.org/intra/aps/documents/aps autoevaluacio_cast_IMP }}$ A5.pdf>

GUTIÉRREZ CRUZ, A., GARCÍA GÁMEZ, E. y GUIJARRO GARVI, M. (2014): Estudios universitarios de posgrado en cooperación internacional para el desarrollo: mapa de competencias formativas, en Actas del II Congreso Internacional de Estudios del Desarrollo. Huelva, del 16 al 18 de junio de 2014. http://www.uhu.es/IICIED/actas.php

GUTIÉRREZ CRUZ, A. (2013). "Repensando la formación en cooperación para el desarrollo desde el enfoque de competencias", en Actas del I Congreso Internacional de Ciencias de la Educación y del Desarrollo. Santander, 10 al 12 de octubre de 2013. http://www.ugr.es/ aepc/WEBEDUCACION/LIBRORESUMENESEDUCACION_.pd $\underline{\mathrm{f}}$

GUTIÉRREZ CRUZ, A. y GUIJARRO GARVI, M. (2013). “Competencias profesionales en cooperación internacional para el desarrollo" en Actas del VI Congreso Universidad y Cooperación al Desarrollo. Valencia, del 24 al 26 de abril de 2013. http://www.sextocongresocud.es/index.php/actas-del-congreso/

GUTIÉRREZ CRUZ, A. (2011). "La formación inicial del cooperante. Aportes desde el enfoque de competencias", en $V$ Congreso Universidad y Cooperación al Desarrollo. Cádiz, del 6 al 8 de abril de 2011. http://ocud.es/sites/default/files/docs/000.pdf

HALSTED, A. (1998). "Educación redefinida: la promesa del Aprendizaje Servicio" en Actas del I Seminario de Aprendizaje Servicio. Ministerio de Cultura y Educación de la Nación, Buenos Aires, pp. 23-32.

MARTÍN GARCÍA, X. et al. (2009). La pedagogía del aprendizaje servicio. Aprendizaje Servicio (APS): educación y compromiso cívico. Barcelona: Graó.

MARTÍNEZ MARTÍN, M. (coord.) (2008). Aprendizaje Servicio y responsabilidad social de las universidades. Barcelona: Octaedro.

MARTÍNEZ ODRÍA, A. (2007). "Service - Learning o Aprendizaje-Servicio. La apertura de la escuela a la comunidad local como propuesta de educación para la ciudadanía” en Bordón, 59 (4), 611-626.

MARTÍNEZ USARRALDE, M.J. (2014). “Otras metodologías son posibles... y necesarias. Cuando la cooperación para el desarrollo en educación encontró al ApS

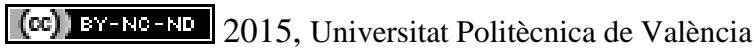

Congreso In-Red (2015) 
Aprendizaje Servicio en el Máster Iberoamericano en Cooperación Internacional y Desarrollo: una experiencia de innovación docente

(Aprendizaje Servicio)" en Universidad y Cooperación al Desarrollo. Contribuciones de las Universidades al Desarrollo Humano. Valencia: Universidad Politécnica de Valencia, p. 135-153.

PUIG, J.M et al. (2009). Aprendizaje Servicio. Educación y Compromiso Cívico. Barcelona: Grao.

PUIG, J., MARTÍN, X. Y BATLLE, R. (2008). Cómo iniciar un proyecto de aprendizaje y servicio solidario. Guía Zerbikas. Bilbao: Fundación Zerbikas.

PUIG, J. M.; BATLLE, R.; BOSCH, C. y PALOS, J. (2007). Aprendizaje servicio. Educar para la ciudadanía. Barcelona, Ministerio de Educación y Ciencia y Octaedro

PUIG ROVIRA, J. M. Y PALOS RODRÍGUEZ, J. (2006). "Rasgos pedagógicos del aprendizaje-servicio” en Cuadernos de Pedagogía, 357, pp. 60-63.

RODRÍGUEZ GALLEGO, M. (2014). "El Aprendizaje Servicio como estrategia metodológica en la Universidad” en Revista Complutense de Educación, 25 (1), p. 95113. 